\title{
Suppression of TLR4-MyD88 signaling pathway attenuated chronic mechanical pain in a rat model of endometriosis
}

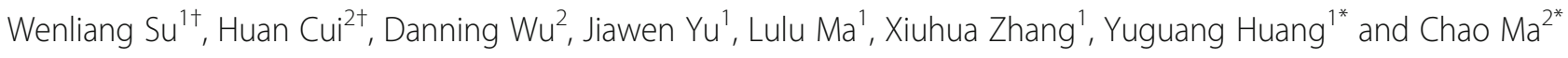

\begin{abstract}
Background: As a classic innate immunity pathway, Toll-like receptor 4 (TLR4) signaling has been intensively investigated for its function of pathogen recognition. The receptor is located not only on immune cells but also on sensory neurons and spinal glia. Recent studies revealed the involvement of neuronal TLR4 in different types of pain. However, the specific role of TLR4 signaling in the pain symptom of endometriosis (EM) remains obscure.

Methods: The rat endometriosis model was established by transplanting uterine horn tissue into gastrocnemius. Western blotting and/or immunofluorescent staining were applied to detect high mobility group box 1 (HMGB1), TLR4, myeloid differentiation factor-88 adaptor protein (MyD88), and nuclear factor kappa-B-p65 (NF-kB-p65) expression, as well as the activation of astrocyte and microglia. The antagonist of TLR4 (LPS-RS-Ultra, LRU) and MyD88 homodimerization inhibitory peptide (MIP) were intrathecally administrated to assess the behavioral effects of blocking TLR4 signaling on endometriosis-related pain.

Results: Mechanical hyperalgesia was observed at the graft site, while HMGB1 was upregulated in the implanted uterine tissue, dorsal root ganglion (DRG), and spinal dorsal horn (SDH). Compared with sham group, upregulated TLR4, MyD88, and phosphorylated NF-KB-p65 were detected in the DRG and SDH in EM rats. The activation of astrocytes and microglia in the SDH was also confirmed in EM rats. Intrathecal application of LRU and MIP alleviated mechanical pain on the graft site of EM rats, with decreased phosphorylation of NF-KB-p65 in the DRG and reduced activation of glia in the SDH.
\end{abstract}

Conclusions: HMGB1-TLR4-MyD88 signaling pathway in the DRG and SDH may involve in endometriosis-related hyperpathia. Blockade of TLR4 and MyD88 might serve as a potential treatment for pain in endometriosis.

Keywords: Endometriosis, Pain, HMGB1, Toll-like receptor 4, Dorsal root ganglion, Spinal dorsal horn

\footnotetext{
* Correspondence: garybeijing@163.com; machao@ibms.cams.cn

${ }^{+}$Wenliang Su and Huan Cui contributed equally to this work.

'Department of Anesthesiology, Peking Union Medical College Hospital,

Chinese Academy of Medical Sciences and Peking Union Medical College,

Beijing, China

2Department of Human Anatomy, Histology and Embryology, Institute of

Basic Medical Sciences, Neuroscience Center, Chinese Academy of Medical Sciences, School of Basic Medicine, Peking Union Medical College, Beijing, China
}

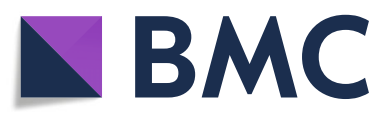

(- The Author(s). 2021 Open Access This article is licensed under a Creative Commons Attribution 4.0 International License, which permits use, sharing, adaptation, distribution and reproduction in any medium or format, as long as you give appropriate credit to the original author(s) and the source, provide a link to the Creative Commons licence, and indicate if changes were made. The images or other third party material in this article are included in the article's Creative Commons licence, unless indicated otherwise in a credit line to the material. If material is not included in the article's Creative Commons licence and your intended use is not permitted by statutory regulation or exceeds the permitted use, you will need to obtain permission directly from the copyright holder. To view a copy of this licence, visit http://creativecommons.org/licenses/by/4.0/. The Creative Commons Public Domain Dedication waiver (http://creativecommons.org/publicdomain/zero/1.0/) applies to the data made available in this article, unless otherwise stated in a credit line to the data. 


\section{Introduction}

Endometriosis (EM), defined as the emergence of endometrial tissue outside the uterus, is a common and costly estrogen-dependent disorder, affecting about 176 million women worldwide [1]. The hallmark symptom of EM is chronic pain, mostly occurring around the ectopic uterine tissue [2, 3]. Classically, the available treatments included pain medications, hormones, surgery, and fertility treatment [4-7]. However, the outcomes were unsatisfying or with intolerable side effects, such as tissue lesions and the maladjustment of estrogen [8-10]. Identifying the pathogenesis of pain in EM and developing effective treatments remain necessary.

High mobility group box 1 (HMGB1), a potent proinflammatory mediator, is an endogenous ligand of TLRs, including TLR2, TLR4, and TLR5, which might lead to allodynia through activating TLR signaling [11]. In addition, HMGB1 served as a potential indicator of fibrosis in ectopic uterine tissue [12], which also had been identified as a promising and admissible biomarker in plasma for EM. Sensory neurons in the DRG, especially the nociceptors, could be facilitated by HMGB1 in an advanced glycosylation end product-specific receptor (RAGE) and transient receptor potential vanilloid-1 (TRPV1) dependent manner [11, 13, 14]. The spinal HMGB1 was also sufficient and necessary for both local and generalized hypersensitivity in the partial infraorbital nerve transection model and contributed to analgesic tolerance under the condition of chronic morphine usage $[15,16]$.

Toll-like receptors (TLRs) function as sensors for external pathogens in innate immunity. TLR4 protects the host cells by recognizing external pathogens and adjusting downstream inflammatory cascades [17]. Recent studies suggested that TLR4 was expressed in smalldiameter primary sensory neurons, which might regulate nociceptive sensation like itch and pain [18-20]. The combination of HMGB1 and TLR4 induced the recruitment of myeloid differentiation factor-88 adaptor protein (MyD88) and NF- $\mathrm{B}$-depended transcriptional process in sensory neurons and glial cells, which was associated with multiple types of pain [21-25]. Nerve injury also elicited intensive neuroinflammation in the DRG through the HMGB1-TLR4-MyD88 signaling pathway, featured by the prominent production of TNF- $\alpha$ and IL-1 3 [26-28]. In the SDH, TLR4-positive astrocytes and microglial cells evoked the central sensitization and promoted the induction and persistence of pain by producing multiple cytokines [29, 30].

Considering the essential role of HMGB1 in EM's pathogenesis, we hypothesized that the HMGB1-TLR4MyD88 signaling pathway in the DRG and SDH was activated, which promoted the development of pain in EM. The suppression of TLR4 and Myd88 could alleviate mechanical pain around the ectopic uterine tissue. We aimed to reveal the changes of HMGB1-TLR4-MyD88 signaling pathway in DRG and SDH in a rat EM model, and the behavioral effects of blocking TLR4 and MyD88 on EM-related pain were also explored. This study might provide potential therapeutic strategies for the pain symptom in EM.

\section{Methods \\ Animals}

Adult female Sprague Dawley rats (180-220 g) were purchased from HFK Bioscience Co., Ltd, Beijing, China. All were housed in a controlled environment (room temperature $21 \pm 4{ }^{\circ} \mathrm{C}$, lights on 07:00-19:00 h, 4-5 rat per cage) with food and water ad libitum. All experimental procedures were approved by the Institutional Animal Care and Use Committee in Chinese Academy of Medical Sciences, Institute of Basic Medical Sciences.

\section{The rat endometriosis model}

The rat model of endometriosis was performed as previously reported [3, 31, 32]. Adult female rats were premedicated with intraperitoneal sodium pentobarbital injection $(40 \mathrm{mg} / \mathrm{kg}$ ) under aseptic conditions. After laparotomy, the abdominal cavity was exposed, and the right uterine horn was identified and isolated. One centimeter segment was excised and placed in a Petri dish containing $0.9 \% \mathrm{NaCl}$. The excised uterine horn was opened longitudinally, and a piece of full-thickness $(3 \times 3 \mathrm{~mm})$ uterine tissue was implanted onto the right gastrocnemius muscle by single stitches. The sham surgery followed the same procedure, but the implant sutured to the gastrocnemius muscle was replaced by a $3 \times 3 \mathrm{~mm}$ peritoneal fat instead of uterine tissue.

\section{Drug delivery}

To complete the intrathecal administration, A PE10 conduit (18 cm in length) was implanted intrathecally under anesthesia with inhaled isoflurane (2.5\%), according to previous reports [33]. Briefly, after making a longitudinal incision $(1 \mathrm{~cm})$ between the L4 and L5 vertebrae, a fine needle was inserted through the dura mater, and then the PE 10 conduit was placed rostrally in the subarachnoid space. The proper intrathecal placement was confirmed by checking for a sudden flicking of tail or hindlimb as well as cerebrospinal fluid outflow. The tip of the conduit was fixed at the cervical region of the rats through a subcutaneous tunnel. Before rats were performed with the model of endometriosis as above, they were injected with $2 \%$ lidocaine $(20 \mu \mathrm{L})$ through the conduit. Only rats whose hind legs were paralyzed in 30 $\mathrm{s}$ after injection and did not recover within $30 \mathrm{~min}$ were included in the experiment. 
LPS-RS-Ultra (LRU, InvivoGen, San Diego, CA, USA; dissolved in endotoxin-free water), a TLR4-specific antagonist, was administered through the PE10 conduit (20 $\mu \mathrm{g}$ in $20 \mu \mathrm{L}$ ). MyD88 homodimerization inhibitory peptide (MIP, Novus Biologicals, CO, USA), a MyD88 inhibitory peptide or control peptide (Novus Biologicals, CO, USA) dissolved in $20 \mu \mathrm{L}$ PBS $(500 \mu \mathrm{M})$ was administered according to previous studies [34].

\section{Behavioral assay}

Muscular tenderness was tested by an electronic pressure measuring instrument, as described previously [31, 32]. Rats were cautiously put into a cylindrical acrylic holder with an adjustable baffle. It was intended for easy exposure of hind limbs and measurement of mechanical pain threshold at the implantation site, namely the belly of the gastrocnemius muscle. Before each behavior test, rats were acclimated in the cylindrical acrylic holder for $30 \mathrm{~min}$. The pressure was applied gradually to the gastrocnemius through a 7-mm-diameter probe; the force of which was assessed by the Compact Digital Force Gauge (Wagner, FDX 10, Shanghai Yuyan Instruments Co., Ltd., China). The mechanical threshold was defined as the force in Newtons, and an acute withdrawal of hind leg was considered as a positive response. The baseline mechanical pain threshold was defined as the mean of 3 readings taken at 5 -min intervals before surgery.

\section{Western blot assay}

After deeply anesthetized with sodium pentobarbital (40 $\mathrm{mg} / \mathrm{kg}$ ) and transcardially perfused with sterile PBS, the L4-L6 spinal dorsal horn (SDH) and dorsal root ganglions (DRGs) were excised and quick-frozen in liquid nitrogen. The whole samples were homogenized in T-PER Tissue Protein Extraction Reagent (Thermo Fisher Scientific, USA) containing Protein Phosphatase Inhibitor (Solarbio, Beijing, China) and phosphatase inhibitor Cocktail (CWbio, Beijing, China). The lysates were then homogenized and centrifuged $\left(12000 \times g\right.$ for $15 \mathrm{~min}$ at $\left.4{ }^{\circ} \mathrm{C}\right)$. The supernatants were collected and denatured, and then separated by SDS-polyacrylamide gel electrophoresis gels and transferred to a PVDF membrane (Thermo Fisher Scientific, USA). The membranes were blocked with $5 \%(\mathrm{w} / \mathrm{v})$ BSA (A8020, Bioss, Beijing, China) for $1 \mathrm{~h}$ at room temperature and incubated with primary antibodies overnight at $4{ }^{\circ} \mathrm{C}$, followed by incubation with corresponding secondary antibody for $1 \mathrm{~h}$ at room temperature (Table 1 lists the primary and secondary antibodies used for western blot analysis). The bands were scanned with Tanon 5800 Luminescent Imaging Workstation (Tanon Science \& Technology Co., Ltd. Shanghai, China) by High-sig ECL Western Blotting Substrate (Tanon, China). The band intensity was measured by Image J software (National Institutes of Health, Bethesda, MD, USA). The ratio of target protein versus $\beta$-actin in each sample, reflected by the ratio of band intensity, was used to compare the relative content of target protein among different samples.

\section{Immunofluorescence staining}

Immunofluorescence was performed as Liu et al. described [34]. Rats were pre-medicated with intraperitoneal sodium pentobarbital injection $(40 \mathrm{mg} / \mathrm{kg})$ under aseptic condition and then transcardially perfused with sterile PBS followed by $4{ }^{\circ} \mathrm{C}$ paraformaldehyde (Sigma, USA). The L4-L6 spinal dorsal horn (SDH) and DRGs were collected, fixed in $4 \%$ paraformaldehyde overnight at $4{ }^{\circ} \mathrm{C}$, and then dehydrated in $30 \%$ sucrose until the tissue settled to the bottom of the EP pipe at $4{ }^{\circ} \mathrm{C}$. Later, tissues were embedded in OCT (Tissue-Tek) and serially sectioned in a cryostat (Leica 2000, Germany) into 15$\mu \mathrm{m}$-thickness slices. After permeabilized in $0.3 \%$ Triton $\mathrm{X}-100$ for $15 \mathrm{~min}$ (DRGs) or $1 \mathrm{~h}(\mathrm{SDH})$ at $37^{\circ} \mathrm{C}$, the tissue sections were blocked with $5 \%$ donkey serum for 1 $\mathrm{h}$, and then bound with the target primary antibodies at $4{ }^{\circ} \mathrm{C}$ overnight in a wet box. Corresponding secondary antibodies were incubated for $1 \mathrm{~h}$ at room temperature (Table 2 lists the primary and secondary antibodies used for immunofluorescence staining analysis). The slides were then washed in PBS and coverslipped by Mounting Medium with DAPI (ZSJB-Bio, Beijing, China). Images were captured by laser confocal microscopic imaging system (FV1000 and Olympus FluoView software, Olympus, Japan). The quantification for immunofluorescence

Table 1 List of primary and secondary antibodies used for western blotting analysis

\begin{tabular}{llllll}
\hline Antibody & Host & Company & Catalog number & Dilution & Incubation conditions \\
\hline HMGB1 & Rabbit & Cell signaling technology & $\# 6893$ & $1: 1000$ & Overnight $4{ }^{\circ} \mathrm{C}$ \\
MyD88 & Rabbit & Abcam & ab2064 & $1: 500$ & Overnight $4{ }^{\circ} \mathrm{C}$ \\
TLR4 & Rabbit & Invitrogen & $48-2300$ & $1: 1000$ & Overnight $4{ }^{\circ} \mathrm{C}$ \\
pNF-KB-p65 & Mouse & Cell signaling technology & $\# 13346$ & $1: 1000$ & Overnight $4{ }^{\circ} \mathrm{C}$ \\
B-actin & Mouse & Cell signaling technology & $\# 3700$ & $1: 2000$ & Overnight $4{ }^{\circ} \mathrm{C}$ \\
Anti-rabbit IgG horseradish peroxidase (HRP) & Goat & ZSGB-BIO & ZDR-5306 & $1: 3000$ & $1 \mathrm{~h} \mathrm{RT}$ \\
Anti-mouse IgG horseradish peroxidase (HRP) & Goat & ZSGB-BIO & ZDR-5307 & $1: 3000$ & $1 \mathrm{hRT}$ \\
\hline
\end{tabular}


Table $\mathbf{2}$ List of primary and secondary antibodies used for immunofluorescence staining

\begin{tabular}{|c|c|c|c|c|c|}
\hline Antibody & Host & Company & Catalog ID & Dilution & Incubation conditions \\
\hline HMGB1 & Rabbit & Cell Signaling Technology & \#6893 & $1: 200$ & Overnight $4{ }^{\circ} \mathrm{C}$ \\
\hline MyD88 & Rabbit & Abcam & ab2064 & $1: 400$ & Overnight $4{ }^{\circ} \mathrm{C}$ \\
\hline TLR4 & Rabbit & Invitrogen & $48-2300$ & $1: 400$ & Overnight $4{ }^{\circ} \mathrm{C}$ \\
\hline TRPV1 & Guinea pig & Abcam & ab10295 & $1: 400$ & Overnight $4{ }^{\circ} \mathrm{C}$ \\
\hline PGP9.5 & Guinea pig & Abcam & ab10410 & $1: 400$ & Overnight $4{ }^{\circ} \mathrm{C}$ \\
\hline pNF-kB-p65 & Rabbit & Abcam & ab86299 & $1: 400$ & Overnight $4{ }^{\circ} \mathrm{C}$ \\
\hline GFAP & Chichen & Abcam & ab4674 & 1:1000 & Overnight $4{ }^{\circ} \mathrm{C}$ \\
\hline CGRP & Goat & Abcam & ab36001 & 1:3000 & Overnight $4{ }^{\circ} \mathrm{C}$ \\
\hline $\mid \mathrm{BA} 1$ & Goat & Abcam & ab5076 & $1: 500$ & Overnight $4{ }^{\circ} \mathrm{C}$ \\
\hline NeuN & Mouse & Abcam & ab104224 & 1:1000 & Overnight $4{ }^{\circ} \mathrm{C}$ \\
\hline Anti-rabbit lgG Alexa Fluor 488 & Donkey & Jackson ImmunoResearch & 711-545-152 & $1: 400$ & $1 \mathrm{~h} \mathrm{RT}$ \\
\hline Anti-Guinea pig IgG Alexa Fluor 594 & Donkey & Jackson ImmunoResearch & 706-585-148 & $1: 400$ & $1 \mathrm{~h} \mathrm{RT}$ \\
\hline Isolectin GS-IB4 Alexa Fluor 594 Conjugate & & Invitrogen & 121413 & $1: 400$ & $1 \mathrm{~h} \mathrm{RT}$ \\
\hline Anti-goat IgG Alexa Fluor 488 & Donkey & Abcam & ab150129 & $1: 400$ & $1 \mathrm{~h} \mathrm{RT}$ \\
\hline Anti-goat lgG Alexa Fluor 594 & Donkey & Jackson ImmunoResearch & 705-585-147 & $1: 400$ & $1 \mathrm{~h} \mathrm{RT}$ \\
\hline
\end{tabular}

staining referred to previous studies [20, 34-36]. Briefly, at least 12 fields from 3 randomly selected sections on each ganglion were examined. More than 100 neuronal somata with nucleus profiles for each condition were counted, and the percentiles of immunopositive neurons were reported. According to the cross-sectional areas (CSA) of soma, neurons were classified as small- (CSA < $\left.636 \mu \mathrm{m}^{2}\right)$, medium- (CSA 637-1431 $\mu^{2}$ ), and largesized neurons $\left(\mathrm{CSA}>1431 \mu^{2}\right)$. Three non-adjacent spinal cord sections were randomly selected from the spinal cord segment (L3-L5) for each rat. Three rats were included for each group. The intensity of GFAP and IBA1 staining was measured with Image J.

\section{Statistical analysis}

Data were presented as group mean and its standard error (mean \pm SEM). Shapiro-Wilk test was applied to determine the normality for parametric test. Student's $t$ test was used to examine differences between two groups, while one-way analysis of variance (ANOVA) followed by the Bonferroni post hoc test was used to examine the difference between multiple groups. For the behavioral test, two-way repeated measures ANOVA was used. In the two-way ANOVA tests, one factor was the time point, and the other was the treatment of the rats (receiving EM surgery or Sham; receiving LRU or Vehicle). If the differences were significant, post hoc Bonferroni's test was applied to compare values at different time points. Categorical data was presented as numbers and percentages and compared by chi-square tests. And all statistical analyses were performed in GraphPad Prism for Windows version 7.0 (GraphPad Software,
Inc., San Diego, CA, USA). A statistically significant difference was defined as a two-sided $p$ value $<0.05$.

\section{Results}

\section{Construction of endometriosis in rats.}

Surgical induction of endometriosis was successfully established in rats, and hyperalgesia was confirmed by behavioral tests. In order to exclude the pain induced by incision, behavioral tests were performed 3 days after surgery. Behavior test showed that rats with uterine grafts developed increased pressuring pain since the third day after operation compared with sham group (surgery with abdominal adipose tissue transplant) or naive group (no surgery) (Fig. 1a). On postoperative day (POD) 7 and 14, dissection of the transplanted tissue revealed cystic lesions with a reddish-brown to amber fluid in endometriosis rats, but not in sham-operated rats (Fig. 1b). HE staining showed that the transplanted uterus was integrated into surrounding muscles tightly with extensive fibroblastic proliferation and pervasive congestion (Fig. 1c). Consistent with the basic pathology and symptoms of endometriosis observed in patients clinically, we successfully established the endometriosis model in rats. To determine the expression of HMGB1 in transplant, western blotting was performed, and the data revealed that HMGB1 was upregulated in the transplant compared with the control uterus horn tissue (Fig. 1d).

Upregulation of HMGB1 in nociceptive pathways after EM The immunofluorescence results showed the expression of HMGB1 in sensory neurons. In addition, we found that the subcellular distribution of HMGB1 shifted from the nucleus to the cytoplasm, which indicated HMGB1 


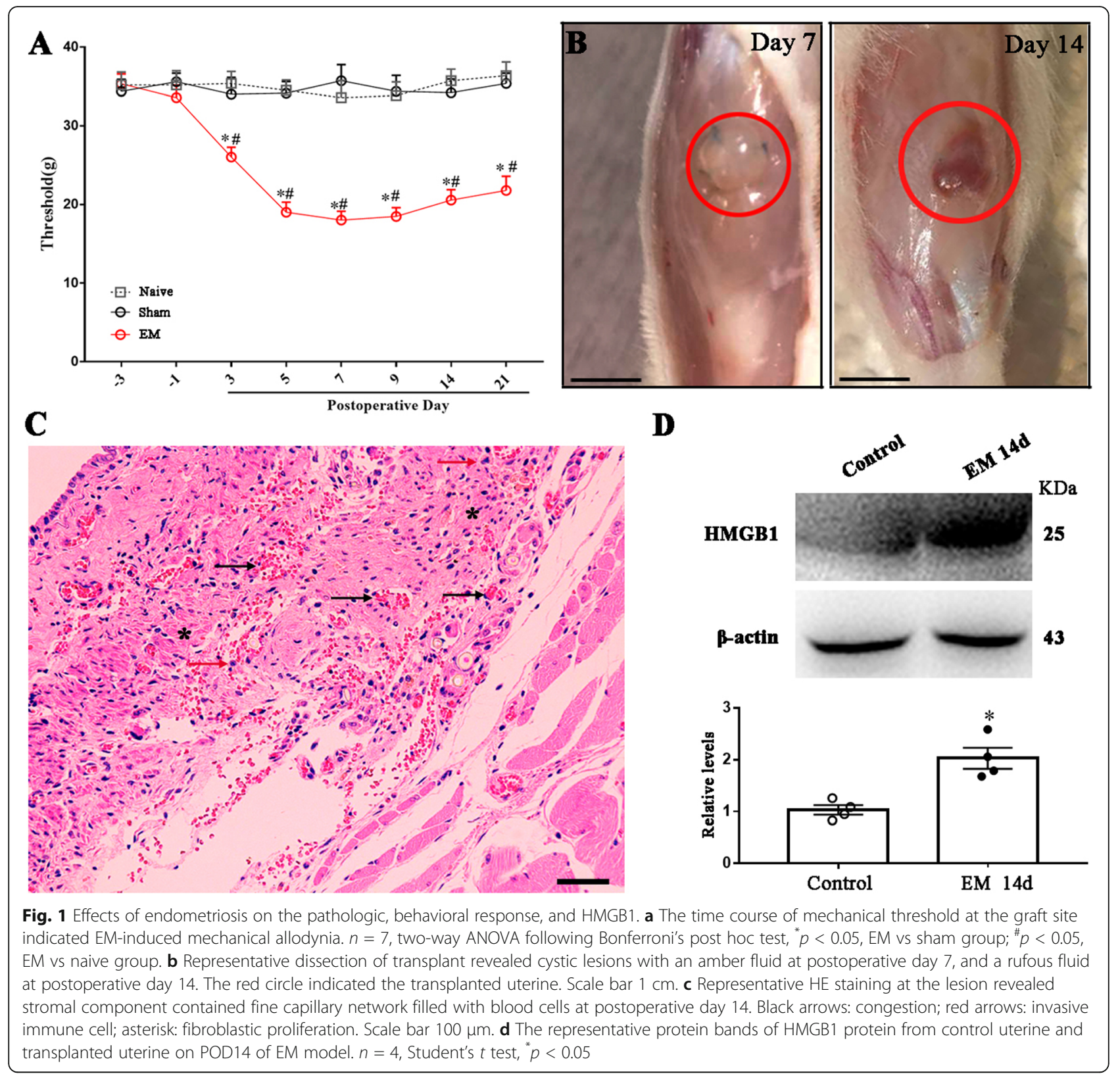

expression in its pro-inflammatory form. Compared with sham group (21.27\%), cytoplasmic HMGB1 was upregulated on POD7 (40.36\%) and POD14 (45.19\%) for EM rats in the dorsal root ganglion (Fig. $2 \mathrm{a}-\mathrm{c}$ and Fig. S1A). On POD14, we determined the subpopulation of HMGB1positive neurons by double-immunofluorescence double staining technique. Summarily, we observed HMGB1 expressed in calcitonin gene-related peptide (CGRP)-positive (39.86\%), non-peptidergic isolectin B4 (IB4)-positive (43.18\%), and transient receptor potential vanilloid-1 (TRPV1)-positive (30.21\%) neurons of DRGs (Fig. 2d-f and Fig. S1B).

According to the immunofluorescence results, the expression of HMGB1 was significantly upregulated in the
SDH of EM rats compared with the sham group (Fig. 2g, $\mathrm{h}$ and Fig. S1C). And HMGB1 protein was mainly distributed in the superficial layers of the SDH. We also found that on POD14, the HMGB1 immunoreactivity was observed in astrocytes (co-localized with GFAP), microglial cells (co-localized with IBA1), and neuronal cells (co-localized with NeuN). These results suggested that EM induced the expression of HMGB1, peripherally and centrally, which might activate the downstream TLR4 signaling pathway (Fig. 2i-k).

\section{Endometriosis upregulated neuronal TLR4 in nociceptors}

First, we determined the time course of TLR4 expression in DRG. The increased expression of TLR4 peaked at 7 


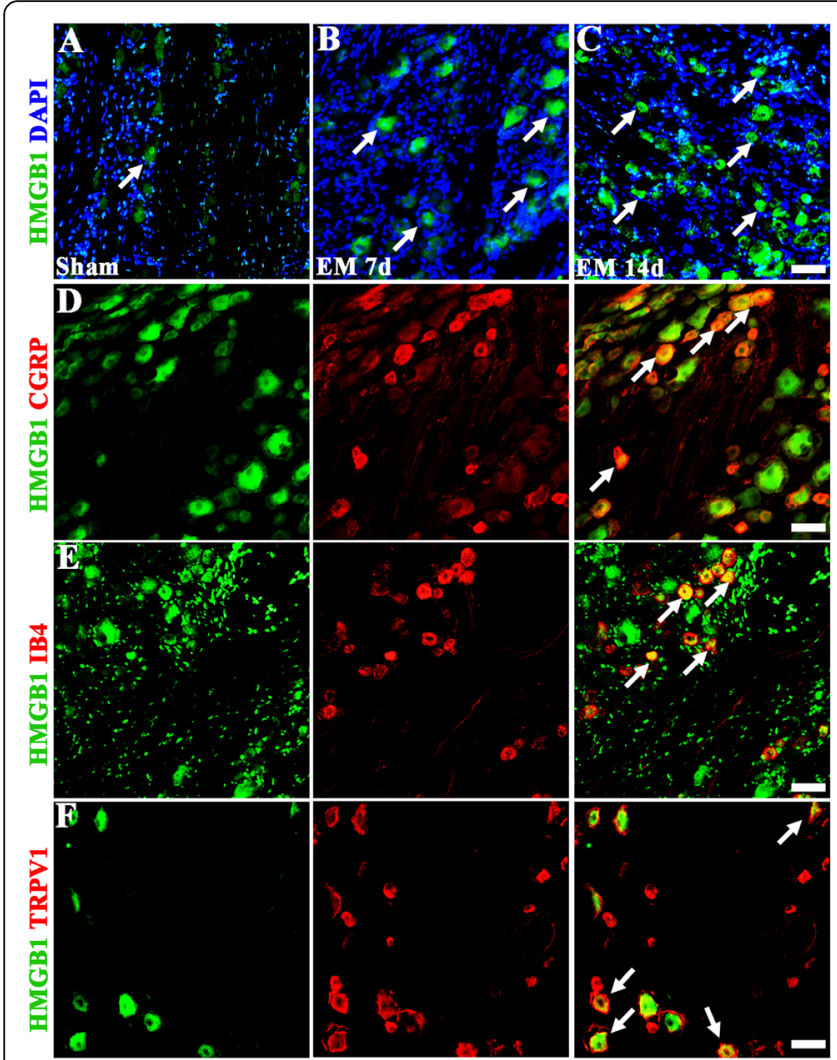

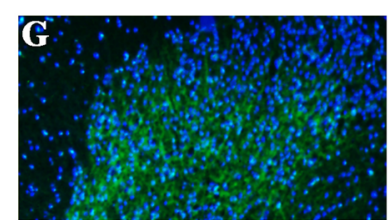

Sham HMGB1 DAPI

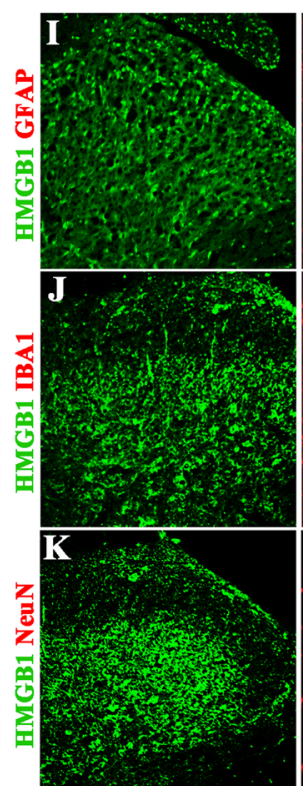

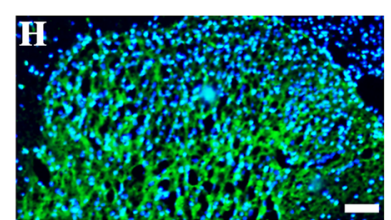

EM 14d HMGB1 DAPI

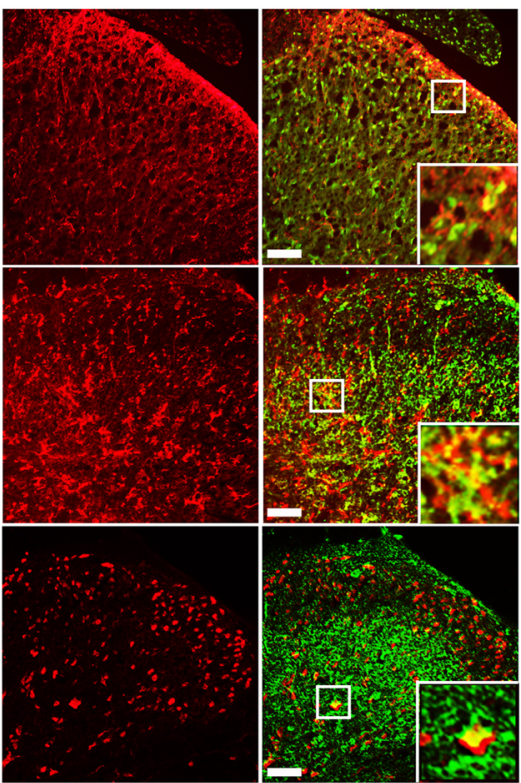

Fig. 2 Endometriosis upregulated HMGB1 in the DRG and SDH. a-c Compared with the sham group, endometriosis upregulated cytoplasmic HMGB1 in sensory neurons in the dorsal root ganglion on POD7 and POD14 after surgery. Scale bar $50 \mu$ m. d-f On POD14 after endometriosis, double immunostaining showed colocalization of HMGB1 with nociceptor markers including CGRP, IB4, and TRPV1, respectively. Scale bar 50 4m. g, h Compared with the sham group, endometriosis upregulated HMGB1 in spinal dorsal horn on POD14 after surgery. Scale bar 50 Hm. i-k On POD14 after endometriosis, immunofluorescence staining showed colocalization of HMGB1 with astrocytic marker GFAP, microglial marker IBA1 and neuronal marker NeuN. Scale bar $50 \mu \mathrm{m}$

days and maintained high throughout the testing periods in EM Group (Fig. 3a, b). Using double immunofluorescent staining, we found that TLR4 was expressed on DRG neurons that were immunopositive for neuron specific marker protein PGP9.5. Immunofluorescence showed TLR4 was upregulated in small-diameter neurons of DRG in EM group compared to sham group (sham 19.84\%, 203/1023; EM 39.39\%, 451/1145). However, there was no difference in TLR4 expression in middle-diameter (sham 13.90\%, 36/259; EM 14.08\%, 39/ 277) and large-diameter neurons (sham 11.40\%, 13/114; EM 12.50\%, 19/152), compared with the sham group (Fig. 3c, d).

In addition, to determine the characteristic of TLR4positive sensory neurons, double immunofluorescence of TLR4 and nociceptive markers, including IB4, CGRP, and TRPV1 was performed. The TLR4 protein immunoreactivity was identified to be distributed in all three categories of nociceptor (Fig. 4a-f). Further comparison revealed that EM upregulated the expression of TLR4 in $\mathrm{IB}^{+}{ }^{+}$(from 18.90 to $36.36 \%$ ), $\mathrm{CGRP}^{+}$(from 23.72 to
64.99\%), and TRPV1 ${ }^{+}$(from 32.12 to $49.77 \%$ ) nociceptors (Fig. 4g).

\section{Endometriosis upregulated neuronal MyD88 in nociceptors}

MyD88, the adaptor of TLR4, has also been found to participate in nociceptive sensation. In this study, we detected the expression of MyD88 in DRG and determined its changes in EM rats. Western blotting results showed that the level of MyD88 was gradually elevated, which peaked on POD14 and maintained high until POD21 (Fig. 5a, b). Immunofluorescence showed that MyD88 also located in sensory neurons, co-localizing with PGP 9.5 in the DRG. Compared with the sham group, the percentage of MyD88-positive neurons in DRG was elevated in the EM group (sham 43.14\%, 365/846; EM 64.44\%, 473/734), specifically in small-diameter neurons (sham 49.00\%, 270/ 551; EM 80.17\%, 384/479) (Fig. 5c, d).

The nociceptive characteristic of ${\mathrm{MyD} 88^{+}}^{+}$sensory neurons was also determined by double immunofluorescence (Fig. 6a-f). The expression of MyD88 was 


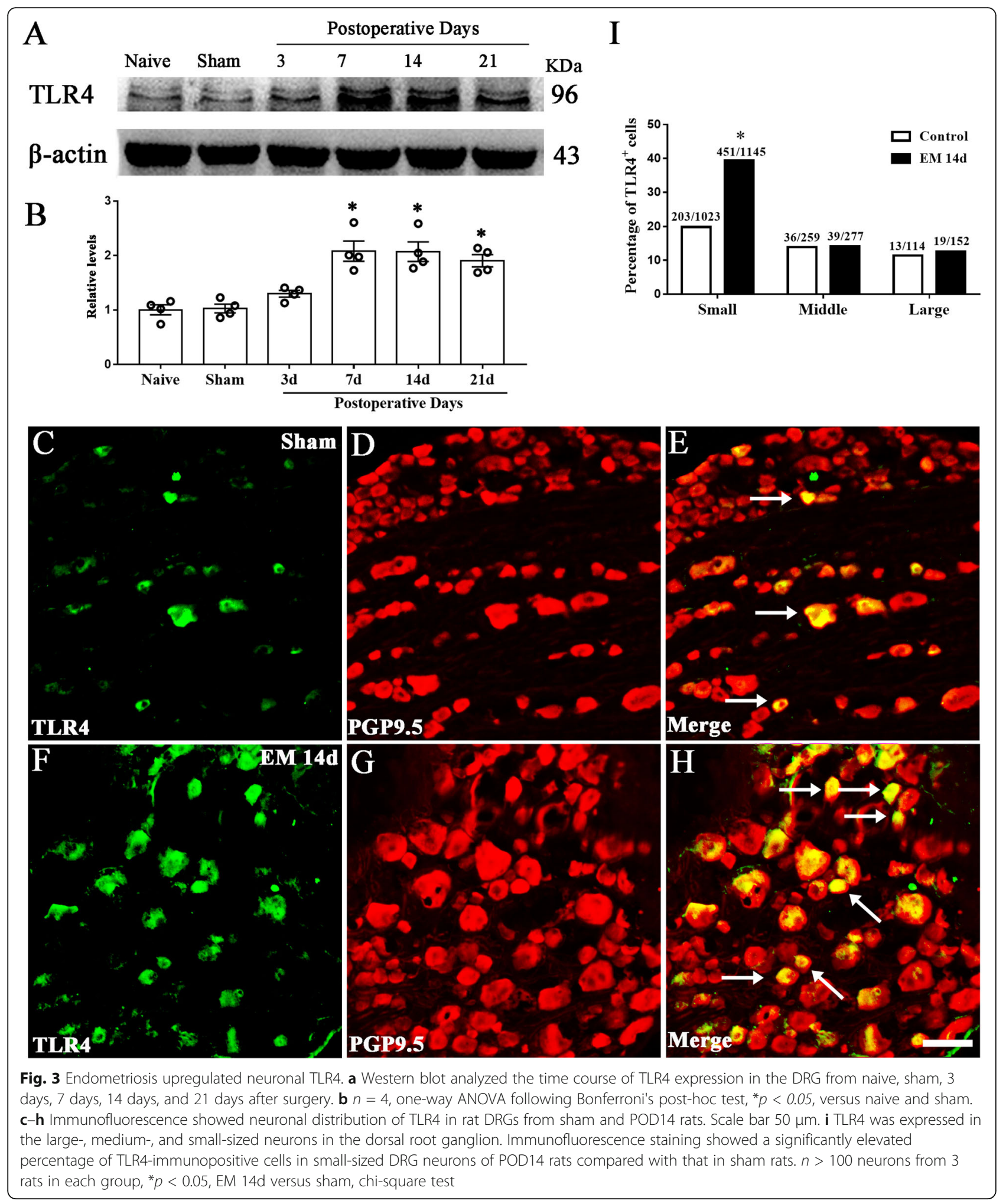

detected in neurons with nociceptive markers including IB4 (sham 19.12\%; EM 48.11\%), CGRP (sham 22.05\%; EM 33.72\%) and TRPV1 (sham 19.77\%; EM 46.27\%), which was upregulated in EM (Fig. 6g). These results showed that TLR4-MyD88 signaling might be activated in nociceptors under the condition of EM. 


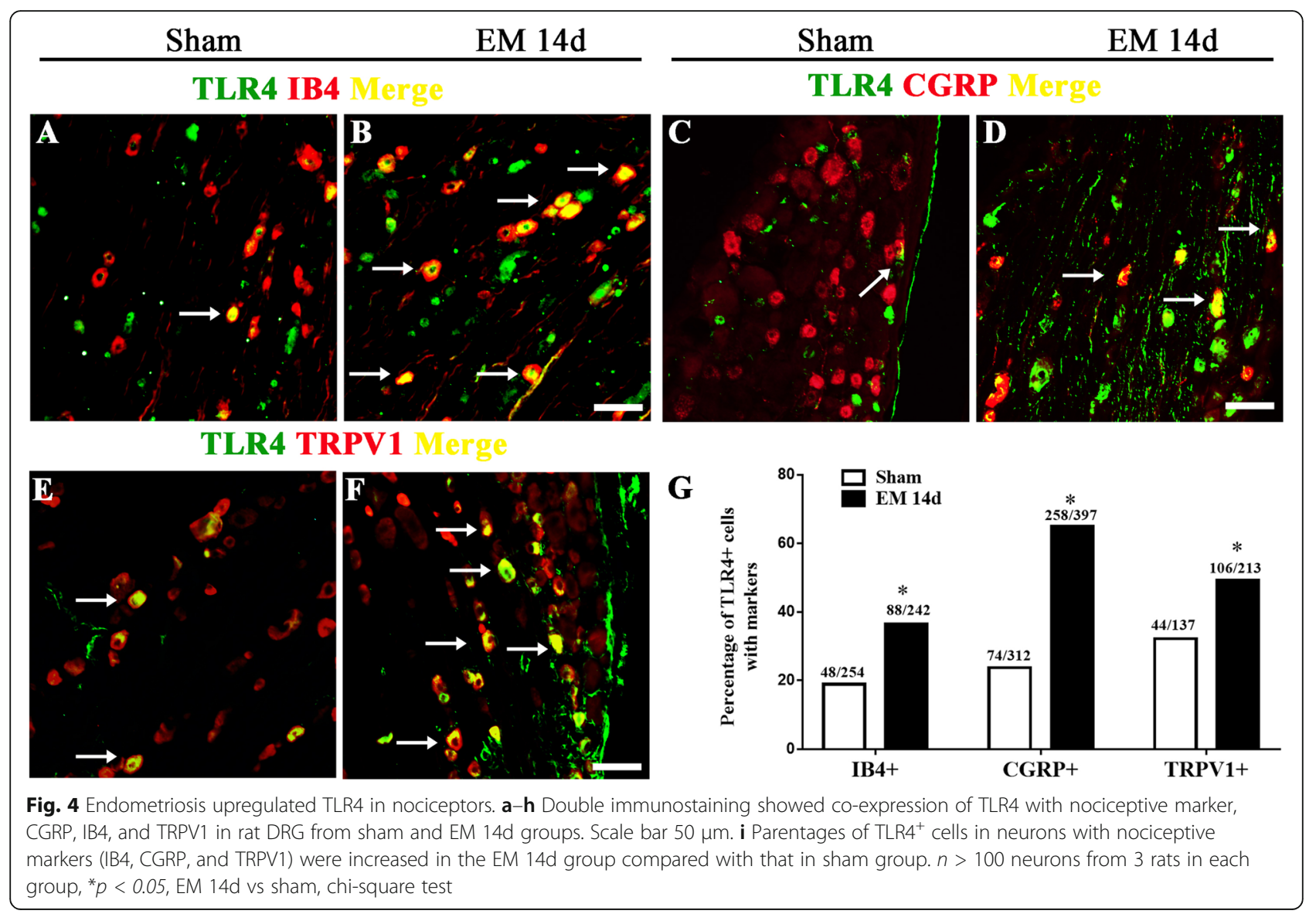

EM induced the activation of NF-KB in sensory neurons $\mathrm{NF}-\kappa \mathrm{B}$ is the transcription factor of the TLR4-MyD88 signaling pathway, which is activated by phosphorylation. Our western blotting results showed that activated phospho-NF-кB p65 increased gradually in DRG from POD3 to POD21, compared with naive and sham groups (Fig. 7a, b). Immunofluorescence showed an elevated expression of phospho-NF-kB p65 in sensory neurons in EM rats (sham 22.93\%, 147/641; EM 56.46\%, 411/728) (Fig. 7c-i). To eliminate the systemic effects on the DRG molecular changes, we compared the expression of HMGB1, TLR4, MyD88, and $\mathrm{p}-\mathrm{NF}-\mathrm{kB}$ in the contralateral and ipsilateral DRG by western blot. Our data showed that these proteins were upregulated in the ipsilateral DRG compared with the contralateral side (Fig. $7 \mathrm{j}-\mathrm{k})$. These results suggested that EM induced the activation of the TLR4/MyD88/NF-kB pathway in the ipsilateral DRG. Besides, the trauma to the uterus in the EM model might impact the pain behavior and signaling pathway in the DRG. In order to determine that the observed behavioral and molecular changes resulted from the EM-pathology, but not the excision of uterine horn tissue, we excided the uterine horn tissue without any transplant. Compared to the control group (opening the abdominal cavity only), the excision of uterine horn tissue did not induce mechanical pain in the musculus gastrocnemius (Fig. S2A). In addition, the excision of the uterine horn did not alter the expression of HMGB1, TLR4, MyD88, and p-NF-kB in the L3-L5 DRGs (Fig. $\mathrm{S} 2 \mathrm{~B}-\mathrm{C})$. The above results suggested that EM-pathology induced mechanical pain in the ectopic site with the activated TLR4-MyD88 pathway in the DRG.

\section{EM activated astrocytes and microglia in SDH}

It has been widely reported that TLR4 signaling evoked extensive activation of glia in the spinal dorsal horn (SDH), which was associated with multiple types of pain. Our previous study also showed that MyD88 was expressed in astrocytes and microglia and participated in neuropathic pain induction and persistence. In this study, western blotting showed that EM persistently upregulated the expression of TLR4 and MyD88 in the spinal dorsal horn from POD3. Increased phosphorylation of NF- $\mathrm{KB}$ p65 also indicated the activation of TLR4 signaling in the spinal dorsal horn of EM rats (Fig. 8a, b). GFAP is the marker for activated astrocytes in the CNS, while IBA1 is considered as an activated marker for activated microglia. Given the observed activation of astrocytes and microglia under multiple pain-related pathologies, we examined astrocytes (GFAP) and 


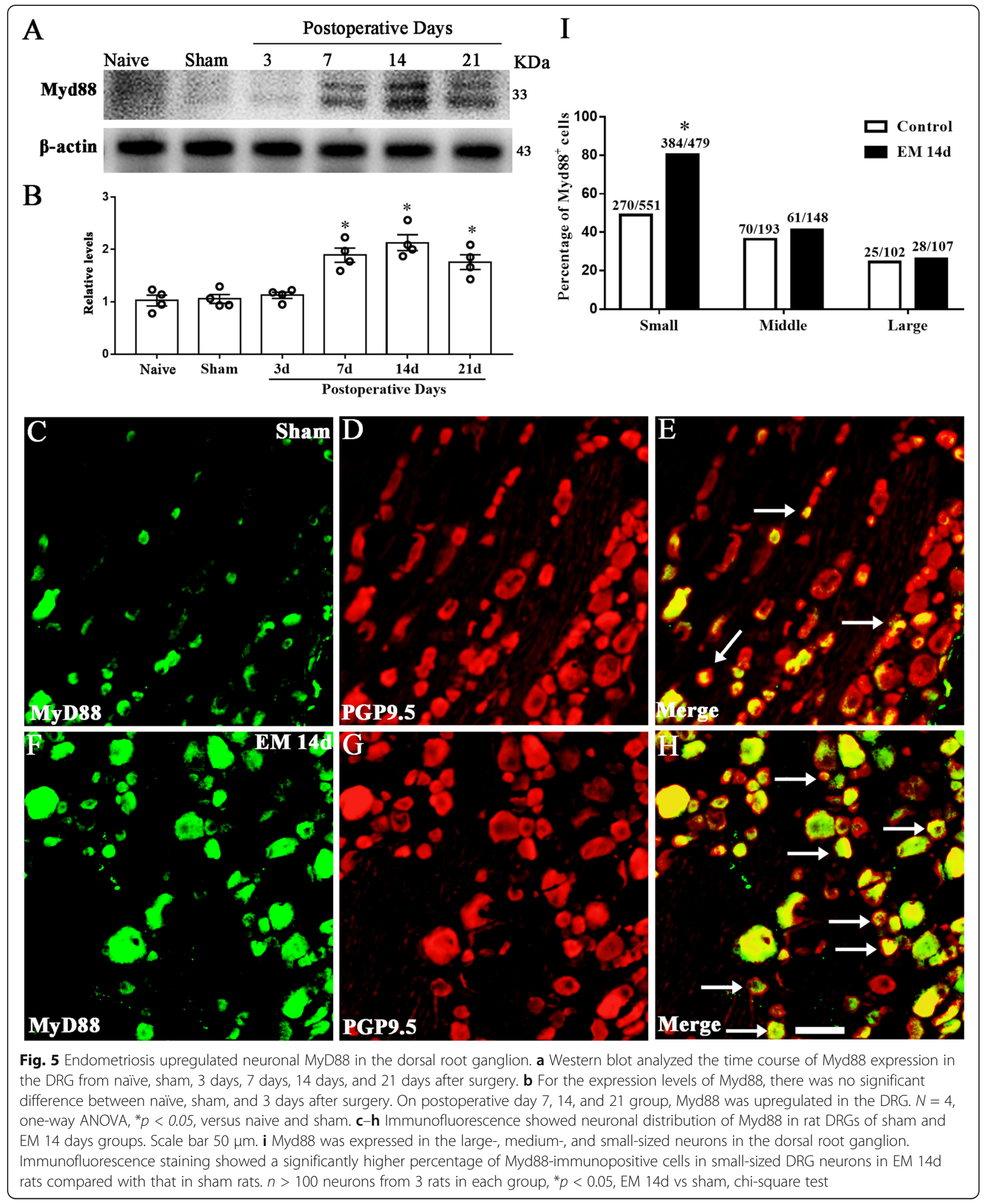

microglia (IBA1) activation by immunofluorescence staining in the SDH. Our immunofluorescence showed that astrocytes and microglia were both activated on ipsilateral (right) SDH compared to contralateral (left) SDH (Fig. 8c-f). In addition, the immunofluorescence staining showed the colocalization of TLR4 with GFAP 


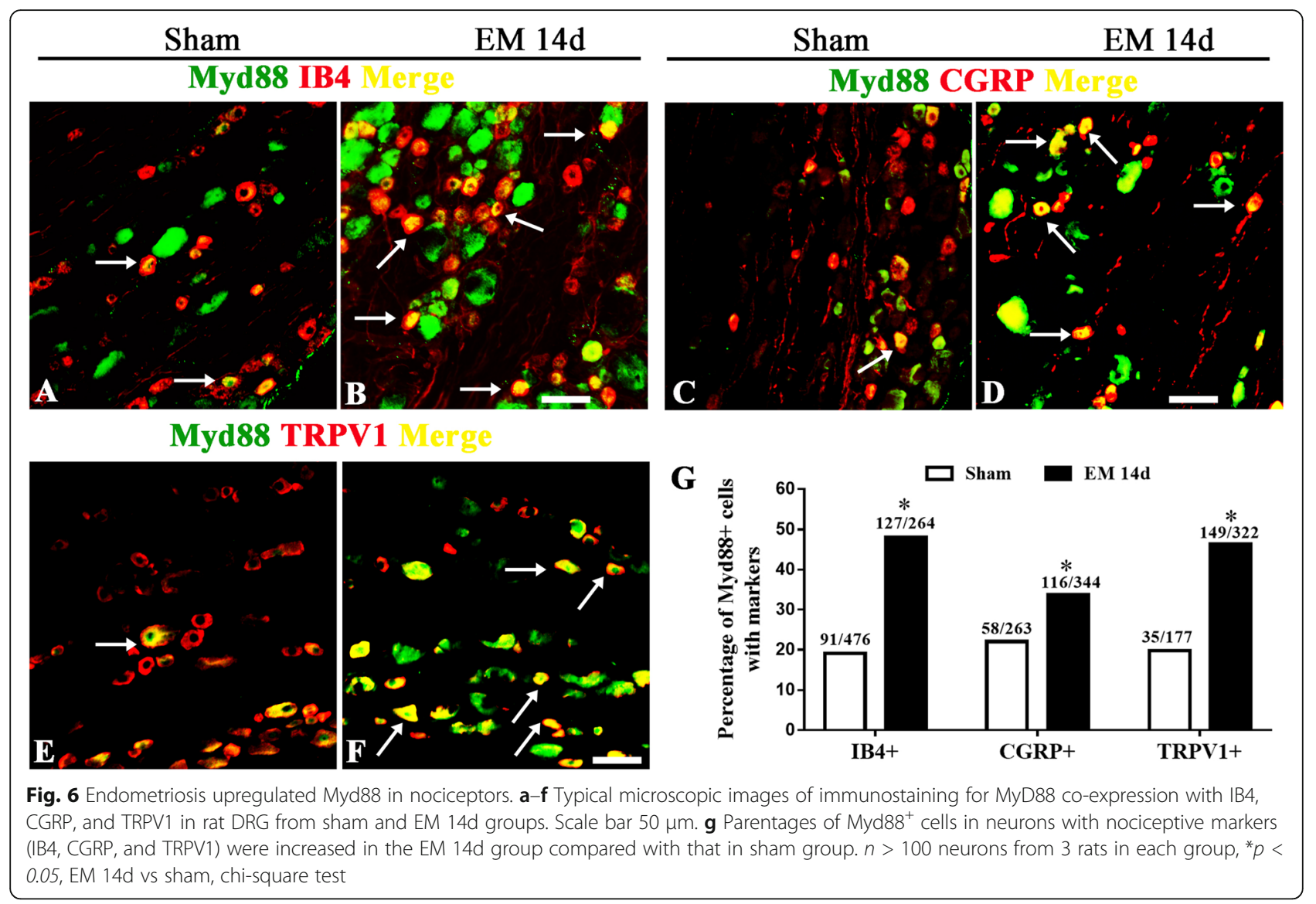

and IBA1 in the SDH of EM rats (POD14), indicating that EM upregulated TLR4 in astrocyte and microglia (Fig. 8g, h).

\section{Blocking TLR4-MyD88 signaling pathway alleviated pain induced by EM}

To measure the behavioral effects of TLR4 signaling on EM-related pain, we blocked the TLR4 signaling pharmacologically and observed behavioral changes. We used the specific TLR4 antagonist (LPS-RS-Ultra, LRU) and MyD88 homodimerization inhibitory peptide (MIP) to inhibit TLR4 and MyD88, respectively. The intrathecal injection was performed to deliver LRU $(20 \mu \mathrm{g}$ in $20 \mu \mathrm{L})$ or MIP $(500 \mu \mathrm{M}$ in $20 \mu \mathrm{l})$ into the lumbar cistern. First, drugs were daily administrated from -3 to -1 day before the surgery. Our data showed that preadministration, both LRU and MIP, alleviated pain in EM rats from POD3 to POD9, while no effect was detected in sham rats. However, both the vehicle of LRU and the control peptide could not relieve the pain in EM rats (Fig. 9a, b). These results indicated that blocking of TLR4 signaling interfered with the development of pain in EM. Moreover, drugs were delivered intrathecally daily for three consecutive days from POD3. We found that both LRU and MIP administration relieved the EM- induced pain with a long-lasting alleviative effect from POD7 to POD21. The buffer for LRU and control peptide of MIP, administrated in the same manner, showed no effect (Fig. 9c, d).

Blocking TLR4-MyD88 signaling pathway suppressed the activation of NF-KB in DRG and activation of glia in SDH under EM

To investigate the mechanism of pain alleviation by LRU and MIP, we examined the expression of phosphorylated NF- $\mathrm{kB}$ p65 in sensory neurons. Western blotting revealed that LRU, applied from POD3 to POD5, decreased the phosphorylation of NF-kB p65 in the DRG on POD14. Meanwhile, MIP treatment, from POD3 to POD5, also decreased the phosphorylation of NF- $\mathrm{kB}$ p65 in the DRG on POD14 (Fig. 10a-d).

Immunofluorescent staining was performed to determine the changes of glial activation after blocking TLR4 signaling in EM rats. Our results showed that both LRU and MIP treatment, applied from POD3 to POD5, decreased the expression of GFAP on POD14, indicating decreased activation of astrocyte (Fig. 11a-d, i). In addition, EM also induced the upregulation of IBA1 and microglial activation on POD14, which could be 


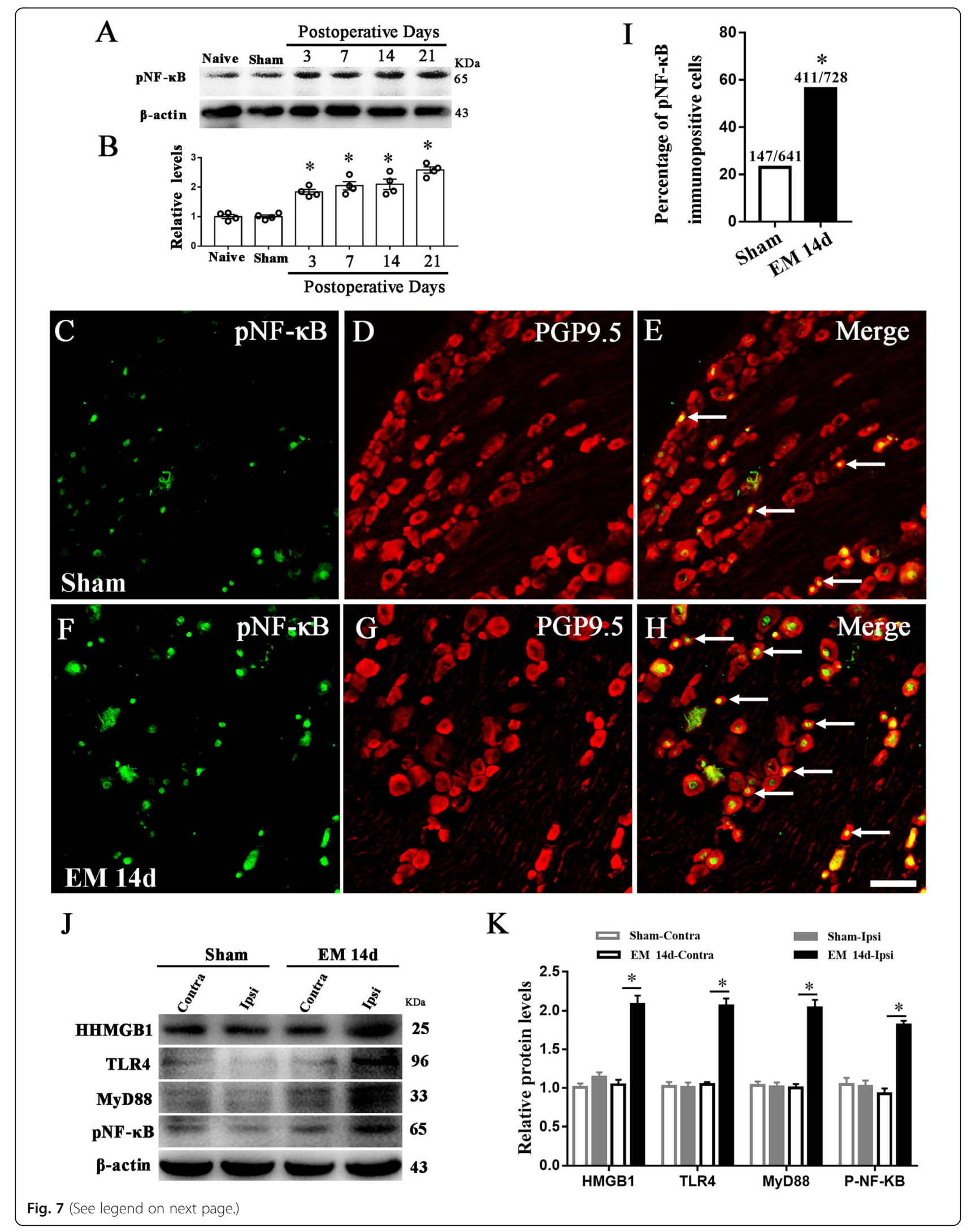


(See figure on previous page.)

Fig. 7 Endometriosis upregulated neuronal pNF-KB-p65 in the dorsal root ganglion. a Western blot analyzed the time course of pNF-KB-p65 expression in the DRG from naïve, sham, 3 days, 7 days, 14 days, and 21 days after EM surgery. $\mathbf{b}$ Western blot revealed that EM upregulated pNF-kB-p65 protein expression in L3-L5 DRGs. $N=4$, one-way ANOVA, ${ }^{*} p<0.05$, versus sham and naïve. $\mathbf{c}-\mathbf{h}$ Immunofluorescence showed neuronal distribution of pNF-KB-p65 in rat DRG of sham and EM 14 days groups. Scale bar $50 \mu \mathrm{m}$. $\mathbf{i}$ Immunofluorescence staining showed a significantly higher percentage of pNF-KB-p65-immunopositive DRG neurons in EM 14d group compared with that in sham group. $n>100$ neurons from 3 rats in each group, ${ }^{*} p<0.05$, EM 14d vs sham, chi-square test. j, k Western blot showed that HMGB1, TLR4, MyD88, and pNF-KBp65 were upregulated in the ipsilateral DRG compared with the contralateral DRG. $N=4,{ }^{*} p<0.05$, Student's $t$ test, EM 14d-Ipsi versus EM 14d-Contra

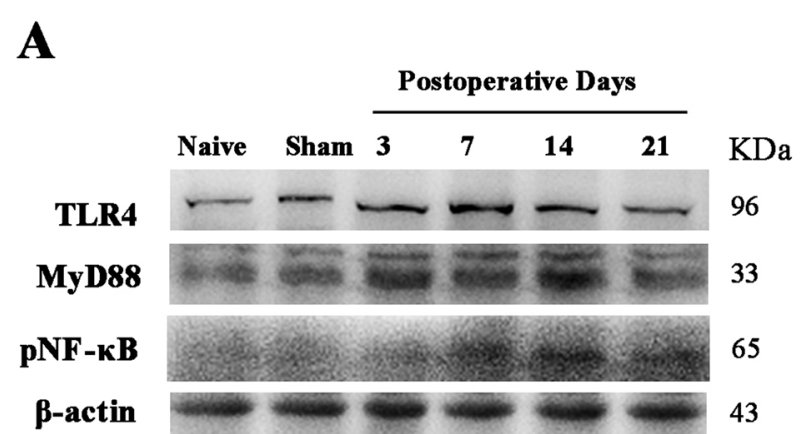

\section{B}
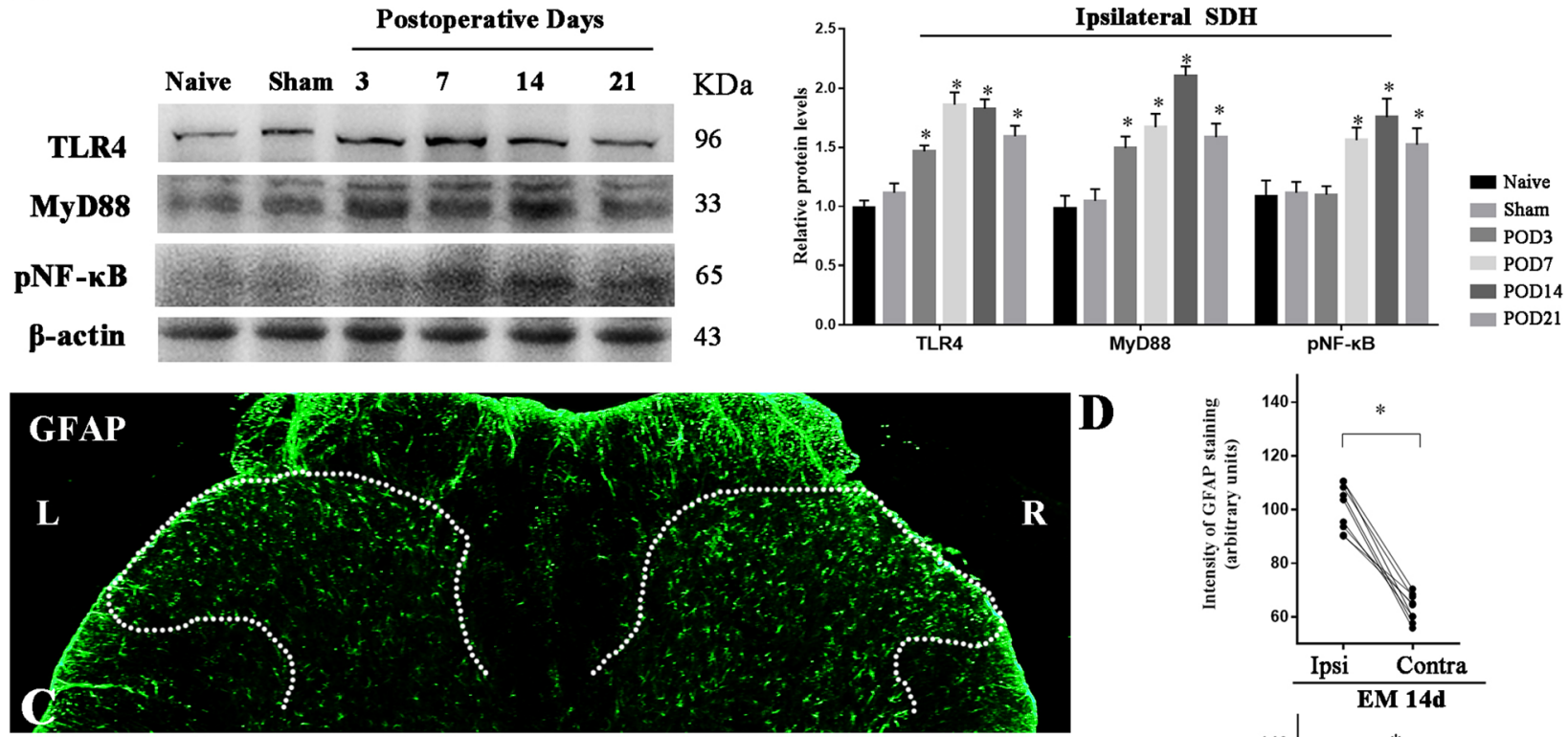

D

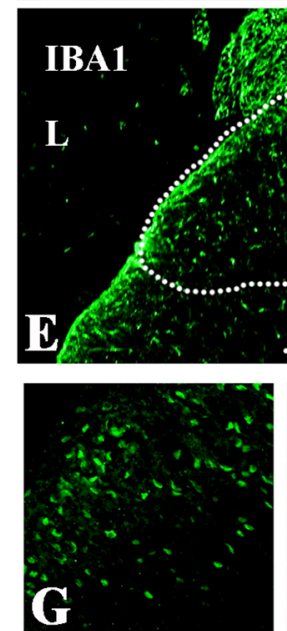

TLR4

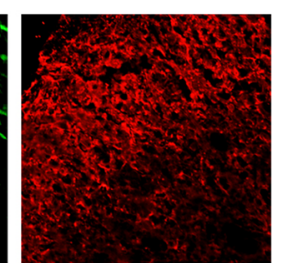

GFAP

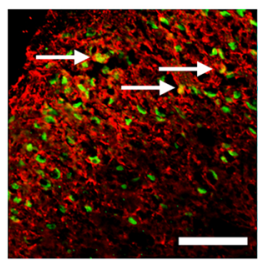

Merge

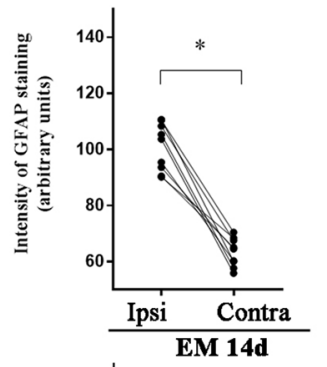

$\mathbf{R}$

Fig. 8 Activation of astrocytes and microglia in ipsilateral SDH of EM rats. a Western blot analyzed the time course of TLR4, MyD88, and pNF-KBp65 expression in ipsilateral SDH after EM. b Quantification of protein levels for TLR4, MyD88, and pNF-kB-p65 expression in ipsilateral SDH. N = 4, ${ }^{*} p<0.05$ versus naive and sham, one-way ANOVA followed by Bonferroni's post hoc test. c-f Representative microscopic images of immunostaining for marker of astrocytes (c, GFAP) and microglia (e, IBA1) in SDH after EM. Scale bar $200 \mu \mathrm{m}$. SDH were indicated by dotted lines. Astrocytes and microglia were significantly activated in the ipsilateral SDH (right) compared to contralateral SDH (left). Fluorescence intensity was applied to measure the relative protein levels. Nine slices from 3 rats in each group, ${ }^{*} p<0.05$, Student's $t$ test. Ipsi: ipsilateral; Contra: contralateral. $\mathbf{g}$ Immunofluorescence staining showed the colocalization of TLR4 and GFAP in the SDH of EM 14d rats. Scale bar $100 \mu \mathrm{m}$. $\mathbf{h}$ Immunofluorescence staining showed the colocalization of TLR4 and IBA1 in the SDH of EM 14d rats. Scale bar $100 \mu \mathrm{m}$ 

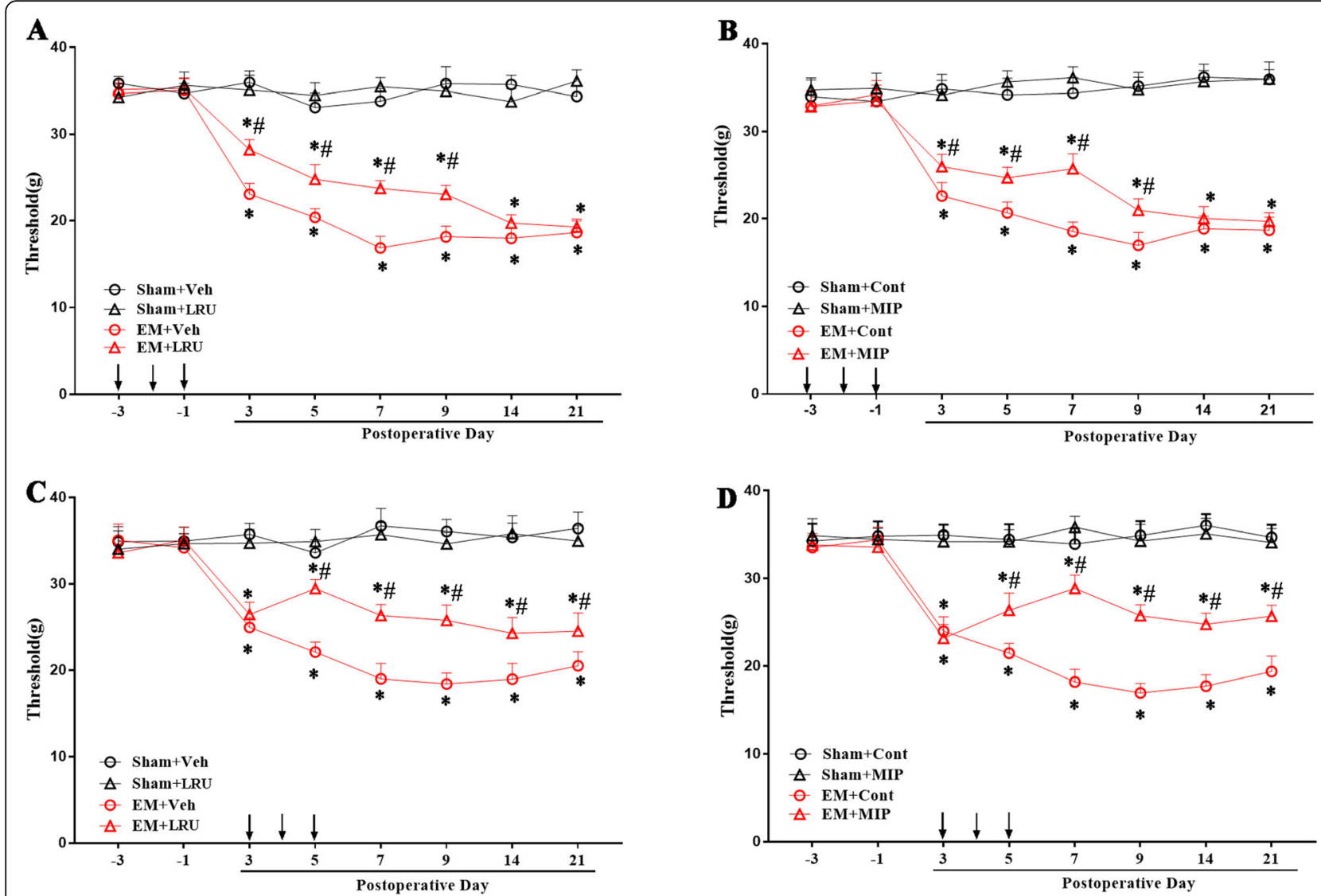

Fig. 9 Attenuated mechanical pain by administration of LRU and MIP for EM rats. $\mathbf{a}$, b Pre-intrathecal injection of LRU and MIP inhibited the development of EM-induced mechanical allodynia. Each administration was indicated by an arrow on the 3, 2, and 1 day before EM surgery. $\mathbf{c}$, $\mathbf{d}$ Mechanical allodynia was alleviated by intrathecal injection of LRU and MIP in the early phase of EM operation. Each administration is indicated by an arrow on the 3, 4, and 5 days after EM surgery. LRU $(20 \mu \mathrm{g})$ was administrated i.t. in a volume of $20 \mu \mathrm{l}$ in endotoxin-free water (Veh). MIP $(500 \mathrm{mM}$ ) was administrated i.t.in a volume of $20 \mu \mathrm{l}$. The control peptide was used in the control (Cont) group. $N=7$ in each group, two-way ANOVA followed by Bonferroni's post hoc test, ${ }^{*} p<0.05 \mathrm{EM}+$ Veh versus sham + Veh and $E M+L R U$ versus sham + LRU (for $\mathbf{a}$ and $\mathbf{c}$ ); $E M+$ Cont versus sham + Cont and EM + MIP versus sham + MIP (for $\mathbf{b}$ and $\mathbf{d}$ ). ${ }^{\#} p<0.05, E M+L R U$ versus EM + Veh (for $\mathbf{a}$ and $\mathbf{c}$ ) and EM + MIP versus $\mathrm{EM}+$ Cont (for $\mathbf{b}$ and $\mathbf{d}$ )

suppressed by intrathecal administration of LRU or MIP from POD3 to POD5 (Fig. 11e-h, j).

\section{Discussion}

In this study, the rat model of endometriosis was performed successfully and exhibited hyperalgesia, determined by anatomical, pathological, and behavioral studies. Not only the implanted uterine tissue, but the DRG and SDH showed a significant upregulation of HMGB1. TLR4 and MyD88 were both upregulated in small-diameter nociceptive neurons in the DRG. Meanwhile, phospho-NF-kB p65, the responsible transcription factor of the TLR4-MyD88 dependent pathway, was upregulated in sensory neurons. We also detected the activation of astrocyte and microglia in the SDH after EM, which might trigger an inflammatory microenvironment and facilitate pain transmission in the SDH. Treatment with TLR4 antagonist (LRU) or MyD88 homodimerization inhibitory peptide (MIP) not only alleviated the hyperpathia in EM, but also reversed the phosphorylation of NF- $\mathrm{kB}$ p 65 in the DRG and the activation of astrocytes and microglia in the SDH. These results suggested that the TLR4-MyD88 signaling pathway in the DRG and SDH, which might be activated by proinflammatory HMGB1, was involved in EM-induced pain.

$\mathrm{EM}$, an estrogen-dependent disorder, is closely related to the estrous cycle, the hallmark symptom of which is chronic pain around the affected region [37]. Surgical induction of endometriosis was first established by Alvarez P. Measurement of hyperalgesia at the site of endometriosis lesion was performed together with the determination of the estrous cycle phase. In this model, pathological and behavioral changes were observed for a considerable period after surgery, regardless of their estrus cycle status. In our study, the EM pathology was determined by characteristic cystic lesions with a reddish- 


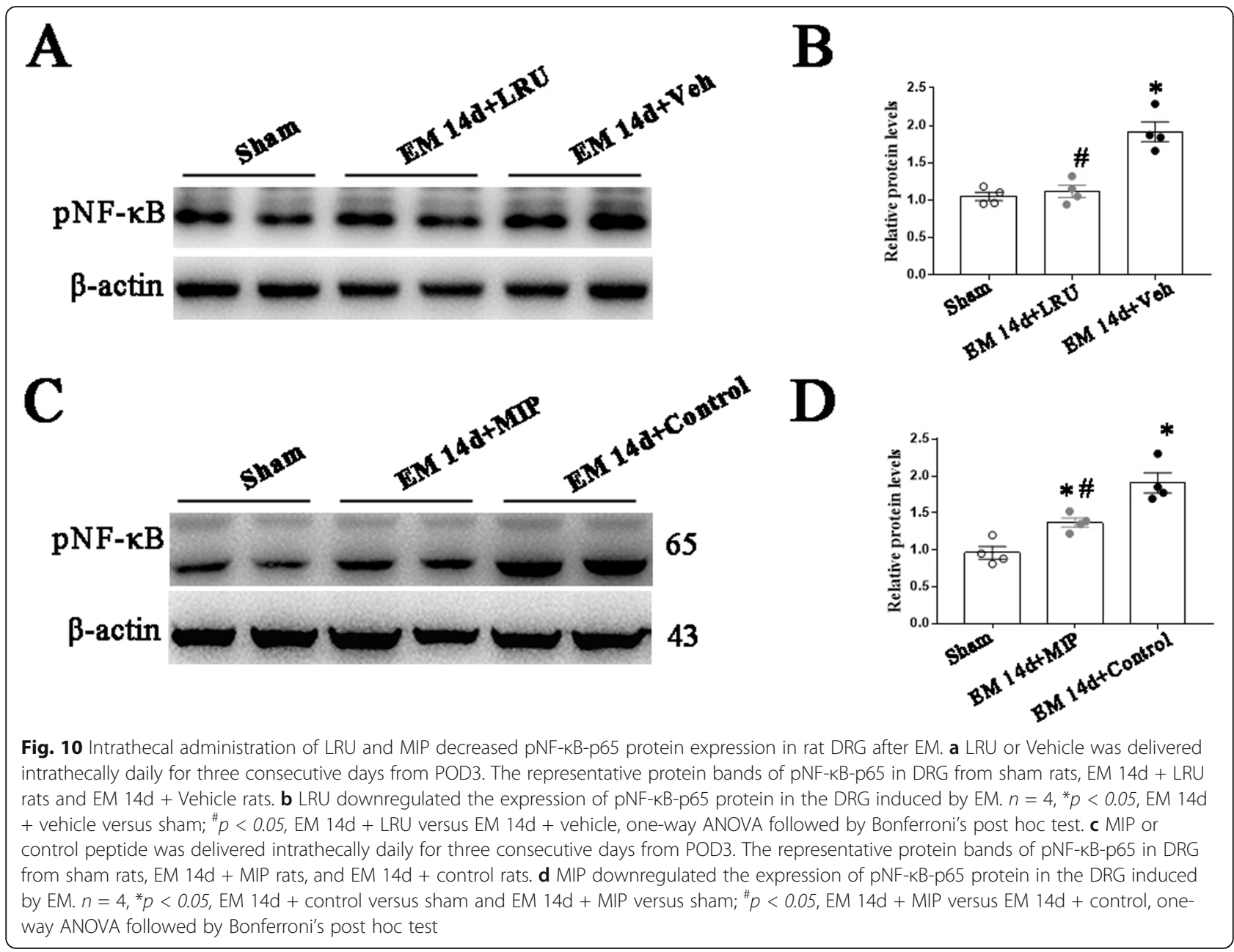

brown to amber fluid in endometriosis rats, which were consistent with previous studies [31, 32, 38]. In addition, HMGB1 was expressed in the transplanted tissue with chronic mechanical pain around the graft site. Previous studies about relieving pain in EM had focused on hormone and reproductive systems from the perspective of gynecology, but the efficacy has been unsatisfactory or with inevitable side effects [9]. Our present study focused on changes in the neuronal system and the neuroimmune communication in the TLR4-MyD88 signaling pathway, finding their possible role in the mechanism of hyperpathia in the rat EM model.

HMGB1, a nuclear non-histone DNA-binding protein, is ubiquitously expressed in multiple cell types, which can be released by necrotic cells during tissue injury in response to inflammatory signals. There are two forms of HMGB1: the nucleus form functions mainly as a transcriptional regulator, and the cytoplasmic form usually elicits the inflammatory process [39]. As a proinflammation mediator, HMGB1 was induced in the DRG and/or $\mathrm{SDH}$ in neuropathic conditions, which also evoked intense cytokines and involved in relative pain symptoms
$[24,40]$. This study verified that neuronal HMGB1 in the DRG and SDH was also induced in the EM model, especially the cytoplasmic HMGB1. The neuronal HMGB1 might be secreted by the relevant cells and elicit following neuroinflammatory cascades through its receptors, including TLR4.

The TLR family plays an essential role in innate immune responses, recognizing external pathogens and protecting host cells through opportune immunoreaction [41]. TLR4, one of the potent HMGB1 receptors, is expressed in DRG, TG, and SDH, facilitating pain and itch sensation [27, 41, 42]. In the DRG, TLR4 was mainly expressed in small-diameter nociceptors, which involved the sensitization of the TRPV1 channel and the persistence of chronic neuropathic pain [22]. TLR4 was also detected in microglia and astrocytes, regulating glial activation and spinal inflammatory microenvironment, which might be responsible for the central sensitization of pain $[43,44]$. Challenged by heat shock protein 90 (HSP90), HMGB1 or LPS, TLR4 could activate both MyD88-dependent and MyD88-independent signaling pathway and mediate the allodynia and 

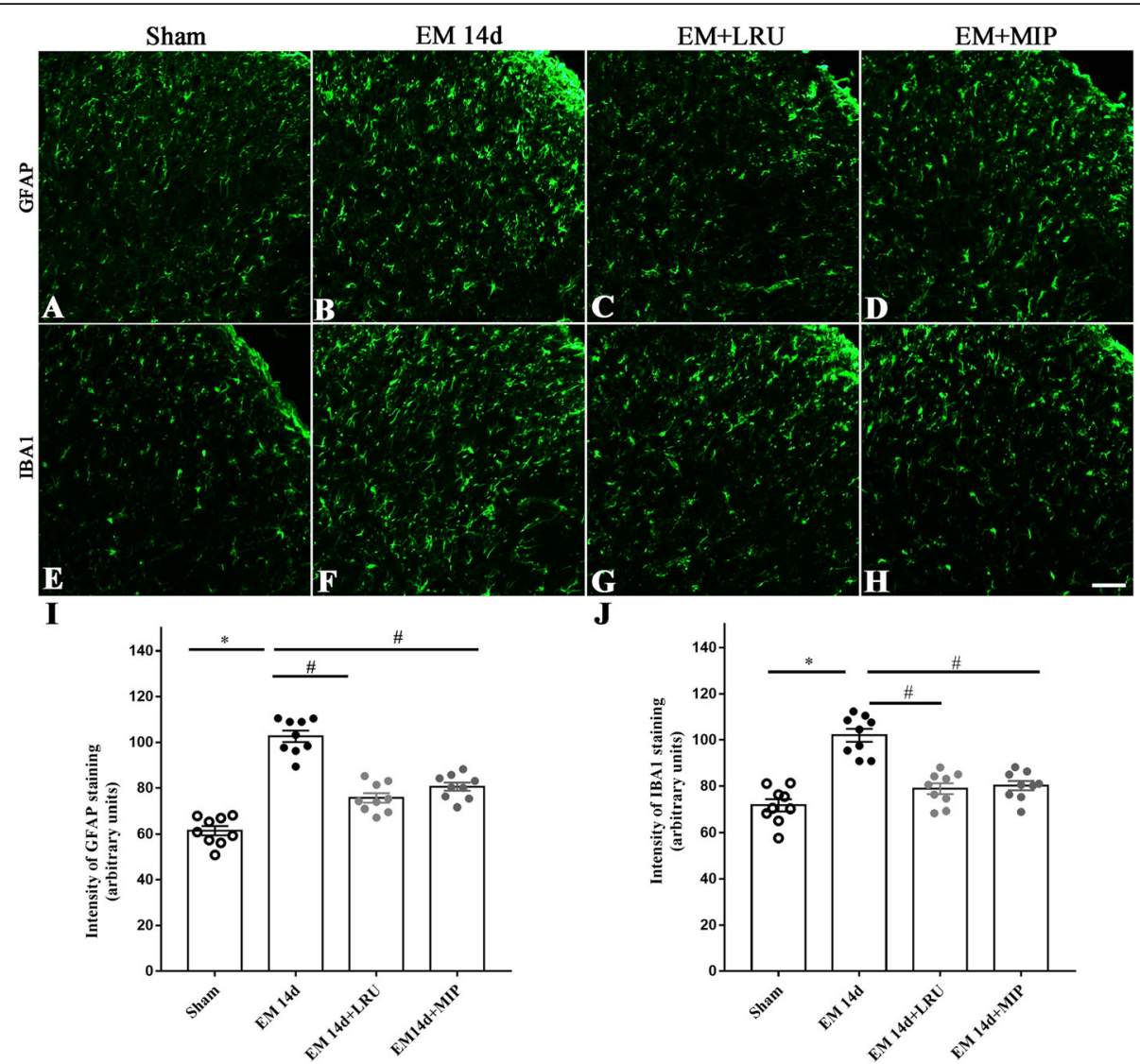

Fig. 11 Attenuated activation of astrocyte and microglia in SDH of EM rats by both LRU and MIP. a-d Representative immunofluorescence images showed inhibitory effects of LRU and MIP on GFAP expression in the SDH of EM rats (14 days after surgery). $\mathbf{e}-\mathbf{h}$ Immunofluorescence showed attenuated IBA1 expression in the SDH of EM rats (14 days after surgery) receiving LRU or MIP. $\mathbf{i}$, $\mathbf{j}$ Quantitative analysis of fluorescence intensity showed that EM upregulated the expression of GFAP and IBA1 in the SDH. MIP and LRU attenuated the expression of GFAP and IBA1 in the SDH of EM rats. Nine slices from 3 rats in each group, ${ }^{*} p<0.05$, EM $14 d$ versus sham group; ${ }^{\#} p<0.05$, EM $14 d$ versus EM $+L R U$ and EM $14 d$ versus EM + MIP group, one-way ANOVA followed by Bonferroni's post hoc test

neuroinflammatory process [41]. MyD88, the adaptor protein for TLR4, also expressed in nociceptors and glial cells and induced glial activation and the production of TNF- $\alpha$ and IL-1 $\beta$, which was related to neuropathic pain [34]. Our data proved the activation of the TLR4Myd88-NF-kB pathway both in DRG and SDH, which might be responsible for the nociceptive facilitation in EM rats. Considering the special role of HMGB1 in the EM pathology, our experiments aimed to reveal the pair of HMGB1 and TLR4 in chronic EM pain. As the manyto-many relationship of the ligands and receptors, the effects of other pairs, such as HMGB1 and RAGE, could be further studied by knockout mice or multiple pharmacological designs.

As TLR4 is not the only receptor for HMGB1, and the TLR4 signaling is conducted by both MyD88-depended and MyD88-independent pathway, TLR4 and MyD88 were blocked respectively to observe the effect of TLR4MyD88 pathway on EM-related pain. We performed pharmacological intervention by intrathecal injection to specifically disturb the TLR4-MyD88 pathway before or after the construction of EM pathology. Preemptively, intrathecal application of LRU or MIP could delay the occurrence of mechanical allodynia. The treatment of LRU or MIP after the formation of initial hyperpathia reversed the pain symptom and showed a long-lasting effect. Consistent with altered pain-related behavior, the phosphorylation of NF-kB-p65 in the DRG and the activation of glia in the SDH was also reversely changed by blocking TLR4-Myd88 signaling in EM rats. By this respective blocking of TLR4 and Myd88 adaptor, our data suggested that the activated TLR4-MyD88 signaling pathway in the DRG and SDH contributed to mechanical pain in EM rats.

\section{Conclusions}

Our study demonstrated that endometriosis induced the expression of HMGB1 in the transplant, dorsal root ganglion, and spinal dorsal horn. Also, endometriosis upregulated the expression of TLR4 and Myd88 in small- 
diameter nociceptors with increased phosphorylation of $\mathrm{NF}-\mathrm{KB}$ in the DRG. In addition, endometriosis-activated astrocytes and microglia with upregulated TLR4-Myd88 signaling in the spinal dorsal horn. Blockade of the TLR4MyD88 signaling pathway attenuated mechanical hyperalgesia and reversed the molecular changes, which implicates this pathway in EM-related pain mechanisms. TLR4MyD88 signaling pathway may play a vital role in EMinduced pain and serve as a potential therapeutic target.

\section{Supplementary Information}

The online version contains supplementary material available at https:/doi. org/10.1186/s12974-020-02066-y.

Additional file 1: Fig. S1 Analysis of HMGB1 expression in DRG and $\mathrm{SDH}$. A. Immunofluorescence staining showed EM significantly upregulated the cytoplasmic HMGB1 in the DRG. $\mathrm{N}=3$, one-way ANOVA, ${ }^{*} P<0.05$ versus sham. B. Percentages of cytoplasmic HMGB1-positive DRG neurons from EM 14d group in neurons with marker of CGRP, IB4 and TRPV1. C. Analysis of staining intensity for HMGB1 indicated the upregulation of $\mathrm{HMGB} 1$ by $\mathrm{EM}$ in the SDH. 9 slices from 3 rats in each group, ${ }^{*} P<0.05$, Student's t-test, EM 14d versus Sham.

Additional file 2: Fig. S2 The excision of uterus horn tissue did not induced mechanical pain at the gastrocnemius muscle. A. The time course of mechanical threshold at the gastrocnemius muscle for rats receiving the excision of uterus horn or not. $\mathrm{N}=8$ in each group, Twoway ANOVA followed by Bonferroni's post-hoc test. B-C. The expression of HMGB1, TLR4, MyD88 and pNF-kB-p65 was not altered by the excision of uterus horn tissue. $\mathrm{N}=4$, One-way ANOVA followed by Bonferroni's post-hoc test.

\section{Abbreviations}

TLR4: Toll-like receptor 4; CGRP: Calcitonin gene-related peptide; CNS: Central nervous system; DRG: Dorsal root ganglion; EM: Endometriosis; GFAP: Glial fibrillary acidic protein; HMGB1: High mobility group box 1; IB4: Isolectin B4; IBA1: Allograft inflammatory factor 1; LRU: LPS-RS-Ultra; MIP: MyD88 homodimerization inhibitory peptide; Myd88: Myeloid differentiation primary response protein 88 ; NF-KB: Transcription factors of the nuclear factor $\mathrm{kB}$; POD: Postoperative day; SDH: Spinal dorsal horn

\section{Acknowledgements}

We thank Dr. Wenyin Qiu, Xiaojin Qian, and Yongmei Chen in the Department of Anatomy, Histology and Embryology, Institute of Basic Medical Sciences, Chinese Academy of Medical Sciences, for their technical assistance in immunohistochemistry.

\section{Availability of data materials}

There is no data, software, databases, and application/tool available apart from the reported in the present study. All data are provided in the manuscript and supplementary data.

\section{Authors' contributions}

Wenliang Su and Huan Cui drafted the manuscript. Wenliang Su performed the immunofluorescence staining and western blotting. Wenliang Su and Huan Cui performed the EM model and behavioral assessment of pain. Danning $\mathrm{Wu}$, Jiawen $\mathrm{Yu}$, and Xiuhua Zhang performed the data analysis. Chao Ma and Yuguang Huang conceived of the study, participated in its design, and helped to draft the manuscript. All authors read and approved the final manuscript.

\section{Funding}

This work was supported by the National Natural Science Foundation of China \#81671098 (Yuguang Huang), the Peking Union Medical College Innovation Fund for Graduates \#2019-1002-65(Wenliang Su) and \#20191001-13(Huan Cui).

\section{Ethics approval and consent to participate}

All animal procedures performed in this study were reviewed and approved by the Institutional Animal Care and Use Committee in Chinese Academy of Medical Sciences, Institute of Basic Medical Sciences, and were conducted in accordance with the guidelines of the International Association for the Study of Pain.

\section{Consent for publication}

Not applicable.

\section{Competing interests}

The authors declare that they have no competing interests.

Received: 1 September 2020 Accepted: 21 December 2020 Published online: 05 March 2021

\section{References}

1. Zondervan KT, Becker CM, Koga K, Missmer SA, Taylor RN, Viganò P. Endometriosis. Nat Rev Dis Primers. 2018;4:9.

2. Peiris AN, Chaljub E, Medlock D. Endometriosis. Jama. 2018;320:26

3. Alvarez P, Chen X, Hendrich J, Irwin JC, Green PG, Giudice LC, Levine JD. Ectopic uterine tissue as a chronic pain generator. Neuroscience. 2012;225: 269-82.

4. Brown J, Crawford TJ, Allen C, Hopewell S, Prentice A. Nonsteroidal antiinflammatory drugs for pain in women with endometriosis. Cochrane Database Syst Rev. 2017;1:Cd0047.

5. Flyckt R, Kim S, Falcone T. Surgical management of endometriosis in patients with chronic pelvic pain. Semin Reprod Med. 2017;35:54-.

6. Diamond MP, Carr B, Dmowski WP, Koltun W, O'Brien C, Jiang P, Burke J, Jimenez R, Garner E, Chwalisz K. Elagolix treatment for endometriosisassociated pain: results from a phase 2, randomized, double-blind, placebocontrolled study. Reprod Sci. 2014;21:363.

7. Brown J, Crawford TJ, Datta S, Prentice A. Oral contraceptives for pain associated with endometriosis. The Cochrane database of systematic reviews. 2018;5:CD001019.

8. Nezhat C, Vang N, Tanaka PP, Nezhat C. Optimal management of endometriosis and pain. Obstetrics and gynecology. 2019;134:834-9.

9. Falcone T, Flyckt R. Clinical Management of Endometriosis. Obstet Gynecol. 2018;131:557-71.

10. Vercellini P, Viganò P, Somigliana E, Fedele L. Endometriosis: pathogenesis and treatment. Nat Rev Endocrinol. 2014;10:261-75.

11. Das N, Dewan V, Grace PM, Gunn RJ, Tamura R, Tzarum N, Watkins LR Wilson IA, Yin H. HMGB1 activates proinflammatory signaling via TLR5 leading to allodynia. Cell reports. 2016:17:1128-40.

12. Cao Y, Liu X, Guo SW. Plasma high mobility group box 1 (HMGB1), osteopontin (OPN), and hyaluronic acid (HA) as admissible biomarkers for endometriosis. Sci Rep. 2019;9:9272.

13. Bestall SM, Hulse RP, Blackley Z, Swift M, Ved N, Paton K, Beazley-Long N, Bates DO, Donaldson LF. Sensory neuronal sensitisation occurs through HMGB-1-RAGE and TRPV1 in high-glucose conditions. J Cell Sci. 2018;131: jcs215939.

14. Allette YM, Due MR, Wilson SM, Feldman P, Ripsch MS, Khanna R, White FA. Identification of a functional interaction of HMGB1 with receptor for advanced glycation end-products in a model of neuropathic pain. Brain, behavior, and immunity. 2014:42:169-77.

15. Hu TT, Yu J, Liu K, Du Y, Qu FH, Guo F, Yu LN, Nishibori M, Chen Z, Zhang $\mathrm{SH}$. A crucial role of HMGB1 in orofacial and widespread pain sensitization following partial infraorbital nerve transection. Brain Behav Immun. 2020;88: 114-24.

16. Qian J, Zhu Y, Bai L, Gao Y, Jiang M, Xing F, Zhang J, Zhao W, Gu H, Mi Y, et al. Chronic morphine-mediated upregulation of high mobility group box 1 in the spinal cord contributes to analgesic tolerance and hyperalgesia in rats. Neurotherapeutics. 2020;17:722-42.

17. Silk E, Zhao H, Weng H, Ma D. The role of extracellular histone in organ injury. Cell death \& disease. 2017;8:e2812.

18. Due MR, Piekarz AD, Wilson N, Feldman P, Ripsch MS, Chavez S, Yin H, Khanna R, White FA. Neuroexcitatory effects of morphine-3-glucuronide are dependent on Toll-like receptor 4 signaling. J Neuroinflammation. 2012;9: 200.

19. Liu XJ, Liu T, Chen G, Wang B, Yu XL, Yin C, Ji RR. TLR signaling adaptor protein MyD88 in primary sensory neurons contributes to persistent 
inflammatory and neuropathic pain and neuroinflammation. Sci Rep. 2016;6: 28188.

20. Liu T, Han Q, Chen G, Huang Y, Zhao LX, Berta T, Gao YJ, Ji RR. Toll-like receptor 4 contributes to chronic itch, alloknesis, and spinal astrocyte activation in male mice. Pain. 2016;157:806-17.

21. Zhou D-M, Zhuang Y, Chen W-J, Li W, Miao B. Effects of duloxetine on the toll-like receptor 4 signaling pathway in spinal dorsal horn in a rat model of diabetic neuropathic pain. Pain medicine (Malden, Mass). 2018;19:580-8.

22. Li Y, Yin C, Li X, Liu B, Wang J, Zheng X, Shao X, Liang Y, Du J, Fang J, Liu B. Electroacupuncture alleviates paclitaxel-induced peripheral neuropathic pain in rats via suppressing TLR4 signaling and TRPV1 upregulation in sensory neurons. Int J Mol Sci. 2019;20:5917.

23. Liu XJ, Zhang Y, Liu T, Xu ZZ, Park CK, Berta T, Jiang D, Ji RR. Nociceptive neurons regulate innate and adaptive immunity and neuropathic pain through MyD88 adapter. Cell Res. 2014;24:1374-7.

24. Gu H, Wang C, Li J, Yang Y, Sun W, Jiang C, Li Y, Ni M, Liu WT, Cheng Z, Hu L. High mobility group box-1-toll-like receptor 4-phosphatidylinositol 3kinase/protein kinase B-mediated generation of matrix metalloproteinase-9 in the dorsal root ganglion promotes chemotherapy-induced peripheral neuropathy. Int J Cancer. 2020;146:2810-21.

25. Walker-Caulfield ME, Guo Y, Johnson RK, McCarthy CB, Fitz-Gibbon PD, Lucchinetti CF, Howe CL. NFkB signaling drives pro-granulocytic astroglial responses to neuromyelitis optica patient lgG. J Neuroinflammation. 2015; 12:185.

26. Wu Z, Wang S, Wu I, Mata M, Fink DJ. Activation of TLR-4 to produce tumour necrosis factor-a in neuropathic pain caused by paclitaxel. European journal of pain (London, England). 2015;19:889-98.

27. Moschetti G, Amodeo G, Paladini MS, Molteni R, Balboni G, Panerai A, Sacerdote P, Franchi S. Prokineticin 2 promotes and sustains neuroinflammation in vincristine treated mice: Focus on pain and emotional like behavior. Brain, behavior, and immunity. 2019;82:422-31.

28. Leung L, Cahill CM. TNF-alpha and neuropathic pain--a review. J Neuroinflammation. 2010;7:27.

29. Xu L, Liu Y, Sun Y, Li H, Mi W, Jiang Y. Analgesic effects of TLR4/NF-kB signaling pathway inhibition on chronic neuropathic pain in rats following chronic constriction injury of the sciatic nerve. Biomedicine \& pharmacotherapy $=$ Biomedecine \& pharmacotherapie. 2018;107:526-33.

30. Stokes JA, Cheung J, Eddinger K, Corr M, Yaksh TL. Toll-like receptor signaling adapter proteins govern spread of neuropathic pain and recovery following nerve injury in male mice. J Neuroinflammation. 2013;10:148.

31. Alvarez P, Bogen O, Levine JD. Role of nociceptor estrogen receptor GPR30 in a rat model of endometriosis pain. Pain. 2014;155:2680-6.

32. Alvarez $\mathrm{P}$, Levine JD. Screening the role of pronociceptive molecules in a rodent model of endometriosis pain. J Pain. 2014;15:726-33.

33. Mazur C, Fitzsimmons B, Kamme F, Nichols B, Powers B, Wancewicz E. Development of a simple, rapid, and robust intrathecal catheterization method in the rat. Journal of neuroscience methods. 2017;280:36-46.

34. Liu F, Wang Z, Qiu Y, Wei M, Li C, Xie Y, Shen L, Huang Y, Ma C. Suppression of MyD88-dependent signaling alleviates neuropathic pain induced by peripheral nerve injury in the rat. J Neuroinflammation. 2017;14: 70 .

35. Jiang H, Shen X, Chen Z, Liu F, Wang T, Xie Y, Ma C. Nociceptive neuronal Fc-gamma receptor I is involved in IgG immune complex induced pain in the rat. Brain Behav Immun. 2017;62:351-61.

36. Liu F, Shen X, Su S, Cui H, Fang Y, Wang T, Zhang L, Huang Y, Ma C. Fcy Receptor I-Coupled Signaling in Peripheral Nociceptors Mediates Joint Pain in a Rat Model of Rheumatoid Arthritis. Arthritis Rheumatol. 2020;72:166878.

37. Chantalat E, Valera M-C, Vaysse C, Noirrit E, Rusidze M, Weyl A, Vergriete $K$ Buscail E, Lluel P, Fontaine $C$, et al. Estrogen receptors and endometriosis. International journal of molecular sciences. 2020;21:2815.

38. Yun BH, Chon SJ, Choi YS, Cho S, Lee BS, Seo SK. Pathophysiology of endometriosis: role of high mobility group box-1 and toll-like receptor 4 developing inflammation in endometrium. PLoS One. 2016;11:e0148165.

39. Thakur V, Sadanandan J, Chattopadhyay M. High-mobility group box 1 protein signaling in painful diabetic neuropathy. Int J Mol Sci. 2020;21:881.

40. Qian J, Zhu Y, Bai L, Gao Y, Jiang M, Xing F, Zhang J, Zhao W, Gu H, Mi Y, et al. Chronic morphine-mediated upregulation of high mobility group box 1 in the spinal cord contributes to analgesic tolerance and hyperalgesia in rats. Neurotherapeutics 2020;17:722-42.
41. Wang $Y$, Zhang $S$, Li H, Wang $H$, Zhang $T$, Hutchinson MR, Yin $H$, Wang $X$. Small-Molecule Modulators of Toll-like Receptors. Acc Chem Res. 2020;53: 1046-55.

42. Adamek P, Heles M, Palecek J. Mechanical allodynia and enhanced responses to capsaicin are mediated by PI3K in a paclitaxel model of peripheral neuropathy. Neuropharmacology. 2019;146:163-74.

43. Freria CM, Bernardes D, Almeida GL, Simões GF, Barbosa GO, Oliveira AL. Impairment of toll-like receptors 2 and 4 leads to compensatory mechanisms after sciatic nerve axotomy. J Neuroinflammation. 2016;13:118.

44. Heiman A, Pallottie A, Heary RF, Elkabes S. Toll-like receptors in central nervous system injury and disease: a focus on the spinal cord. Brain Behav Immun. 2014;42:232-45.

\section{Publisher's Note}

Springer Nature remains neutral with regard to jurisdictional claims in published maps and institutional affiliations.

\section{Ready to submit your research? Choose BMC and benefit from:}

- fast, convenient online submission

- thorough peer review by experienced researchers in your field

- rapid publication on acceptance

- support for research data, including large and complex data types

- gold Open Access which fosters wider collaboration and increased citations

- maximum visibility for your research: over $100 \mathrm{M}$ website views per year

At BMC, research is always in progress.

Learn more biomedcentral.com/submissions 